This item was submitted to Loughborough's Research Repository by the author.

Items in Figshare are protected by copyright, with all rights reserved, unless otherwise indicated.

\title{
Muscle pain from an intramuscular injection of hypertonic saline increases variability in knee extensor torque reproduction
}

PLEASE CITE THE PUBLISHED VERSION

https://doi.org/10.1152/japplphysiol.00139.2020

\section{PUBLISHER}

American Physiological Society

VERSION

AM (Accepted Manuscript)

\section{PUBLISHER STATEMENT}

This paper was accepted for publication in the journal Journal of Applied Physiology and the definitive published version is available at https://doi.org/10.1152/japplphysiol.00139.2020

LICENCE

CC BY-NC-ND 4.0

\section{REPOSITORY RECORD}

Smith, Samuel Andrew, Dominic Micklewright, Sam Winter, and Alexis R Mauger. 2021. "Muscle Pain from an Intramuscular Injection of Hypertonic Saline Increases Variability in Knee Extensor Torque Reproduction". Loughborough University. https://hdl.handle.net/2134/13207607.v1. 


\section{MUSCLE PAIN FROM AN INTRAMUSCULAR INJECTION OF HYPERTONIC} SALINE INCREASES VARIABILITY IN KNEE EXTENSOR TORQUE REPRODUCTION

(1)

Samuel A Smith ${ }^{1}$, Dominic Micklewright ${ }^{2}$, Samantha L Winter ${ }^{1,3}$, Alexis R Mauger ${ }^{1}$ (1)

${ }^{1}$ Endurance Research Group, School of Sport and Exercise Sciences, University of Kent, Chatham Maritime, (UK).

${ }^{2}$ School of Sport, Rehabilitation and Exercise Sciences, University of Essex, Wivenhoe Park, Colchester, (UK).

${ }^{2}$ School of Sport, Exercise and Health Sciences, Loughborough University, Ashby Road Loughborough, (UK).

\section{Author Contributions}

SAS and ARM were responsible for the conception and design of the study, and data acquisition. SAS, DM, SLW, and ARM were responsible for data analysis and interpretation. SAS was responsible for drafting the manuscript. SAS, DM, SLW and ARM were responsible for critically revising and editing intellectual content.

Running head: Muscle pain increases variability in torque reproduction

Correspondence to: Alexis (Lex) Mauger, School of Sport and Exercise Sciences, University of Kent, ME4 4AG, United Kingdom Tel: +44 (0)1634 888997 Email: lex.mauger@gmail.com Institutional URL: https://www.kent.ac.uk/sport-sciences/people/2190/mauger-lex 


\section{ABSTRACT}

Purpose: The intensity of exercise-induced pain (EIP) reflects the metabolic environment in the exercising muscle, so during endurance exercise this may inform the intelligent regulation of work rate. Conversely, the acute debilitating effects of EIP on motor unit recruitment could impair the estimation of force produced by the muscle and impair judgement of current exercise intensity. This study investigated whether muscle pain that feels like EIP, administered via intramuscular injection of hypertonic saline, interferes with the ability to accurately reproduce torque in a muscle group relevant to locomotive exercise. Methods: On separate days, fourteen participants completed an isometric torque reproduction task of the knee extensors. Participants were required to produce torque at 15 and $20 \%$ maximal voluntary torque (MVIT), without visual feedback before (Baseline), during (Pain/No Pain), and after (Recovery) an injection of $0.9 \%$ isotonic saline (Control) or 5.8\% hypertonic saline (Experimental) into the vastus lateralis of the right leg. Results: An elevated reported intensity of pain, and a significantly increased variance in mean contraction torque at both $15 \%(P=0.049)$ and 20\% ( $P=0.002)$ MVIT was observed in the Experimental compared to the Control condition. Both 15 and $20 \%$ target torques were performed at a similar pain intensity in the Experimental condition (15\% MVIT, $4.2 \pm 1.9 ; 20 \%$ MVIT, $4.5 \pm 2.2 ; P>0.05$ ).

Conclusion: These findings demonstrate that the increased muscle pain from the injection of hypertonic saline impeded accurate reproduction of knee extensor torque. These findings have implications for the detrimental impact of EIP on exercise regulation and endurance performance. 


\section{$51 \quad$ New \& Noteworthy}

52 We provide novel data demonstrating that the presence of muscle pain interferes with

53 estimations of torque produced by the knee extensors, which could impair judgement of

54 work-rate during endurance exercise. The novelty of our study is in the application of the

55 hypertonic saline experimental model into a quadriceps muscle during short, submaximal

56 isometric contractions at an intensity that provides a more translatable assessment of the

57 impact of exercise-induced pain on work-rate regulation during whole-body exercise.

58

59 Key words: Nociception, Exercise Regulation, Proprioception, Effort perception, Pain

60

61

62

63

64

65

66

67

68

69

70

71

72

73

74

75 
INTRODUCTION

77

Exercise-induced pain (EIP) increases linearly with exercise intensity and duration (9), and has been suggested to provide useful sensory feedback about the relative strain of exercising muscles $(7,27,31)$. During intense and fatiguing muscle contractions, nociceptors of Group III and IV muscle afferents become sensitised and activated by an accumulation of metabolites which induce the perception of EIP, but are also implicated in peripheral fatigue and the description of its perception $(31,38)$. Resultantly, EIP is often associated with other physiological and psychological factors of fatigue, and has been suggested to independently exacerbate or contribute to the development of fatigue (27). A change in muscle torque complexity, which is suggested to reflect the adaptability of the neuromuscular system and is reduced during fatiguing maximal and submaximal isometric contractions (34), could provide a non-invasive method to evaluate the fatiguing effect of EIP.

During whole-body exercise, sensations of EIP may facilitate conscious control of homeostatic disturbance during exercise by enabling the intelligent regulation of available energetic resources (i.e. pacing) $(12,27,54)$. However, the relationship between EIP and fatigue is likely more complex since it also causes various acute debilitating effects associated with motor unit recruitment (17) and, as a protective mechanism, restricts movement to reduce pain. Consequentially, whilst EIP may provide insight about the metabolic environment in the exercising muscle, these potentially detrimental adaptations may reduce the accuracy of estimations of work done and/or force applied by the muscle, which could impair pacing decisions during whole-body exercise.

Supressing the unpleasant sensations associated with intense exercise may allow a higher exercise-intensity to be tolerated and sustained (28), however near complete removal of this 
information via spinal afferent blockade appears to impair the exerciser's ability to select and maintain a physiologically optimal work rate (3). Spinal blockade studies show the importance of Group III and IV afferents to the performance of whole-body exercise $(2,3)$ but reveal less about the parallel effects of nociception and perceived pain on other systems such as cardiovascular control.

Intramuscular hypertonic saline injection produces a muscle pain that feels like the naturally occurring EIP experienced during intense exercise $(16,50)$, and is therefore a useful method to investigate how EIP affects self-regulation of exercise intensity. This technique has previously been used in contralateral limb-matching tasks to assess the impact of tonic muscle pain on the judgement of torque in small muscle groups $(40,41,57)$. In these studies, increased pain impeded the ability to accurately match torque, with pain intensity and degree of error correlating such that participants consistently overestimated the force generated by the painful muscle.

This experimental approach could, however, be confounded by potential differences between the contralateral limbs $(1,36)$. To provide a more translatable assessment of the impact of EIP on whole-body exercise, the relationship between muscle pain and the reproduction of isometric torque production should be evaluated in the larger muscle groups of the lower limb such as the knee extensors, which have an important and fundamental role in the generation of force during locomotion and exercise.

As such, the aim of the present study was to ascertain whether experimentally induced muscle pain in the vastus lateralis (VL) using an intramuscular injection of hypertonic saline would affect the ability to accurately gauge the torque produced by the knee extensor muscles 
in a single-limb isometric torque reproduction task. We tested the hypothesis that

127

128 experimental muscle pain in the VL reduces torque reproduction accuracy (as quantified by the variance in mismatch between target and actual torque) of low intensity isometric contractions when compared to a placebo control condition.

\section{METHODS}

\section{Ethical Approval}

All procedures and protocols were approved by the School of Sport and Exercises (University of Kent) Research Ethics Advisory Group (Prop 140_2016_17) in conformity with the Declaration of Helsinki, and its later amendments or comparable ethical standards. All participants were informed of the study experimental procedures, and written informed consent was obtained to confirm participation.

\section{Participants}

Fourteen healthy and recreationally active participants (13 male, 1 female; mean \pm SD: age, $25.3 \pm 4.5$ years; height $1.78 \pm 0.1 \mathrm{~m}$; body mass $73.9 \pm 12.3 \mathrm{~kg}$; physical activity $5.6 \pm 2.2$ hours per week) volunteered to participate in the present study. Assuming a statistical power of 0.8 at an alpha level of 0.05 , the sample size was estimated using $G^{*}$ Power software (13) based on the effect size reported in a similar study in our laboratory using hypertonic saline injections (50). All participants attended each visit in a similar psychological state as assessed by the Positive and Negative Affect Schedule (PANAS) (56), which was completed at the start of each visit.

Before each visit, participants were instructed to refrain from vigorous exercise (24 h) and abstain from the consumption of alcohol (48 h), analgesics (6h) and caffeine ( $8 \mathrm{~h})$. 
151 Participants with existing knee pain, cardiorespiratory disease, neurological disorders, blood

152 borne viruses, sore deep tissues, phobia to needles and any allergy were excluded from the 153 study.

\section{Experimental design}

156 In a two-way repeated-measures experimental design, participants performed an isometric 157 torque matching and reproduction task with either pain (a single intramuscular injection of hypertonic saline) or a placebo control (a single intramuscular injection of isotonic saline)

159 (condition factor). Participants attended a familiarisation session, and then completed the 160 experimental conditions in a randomised and counterbalanced order, with each visit separated by a minimum of seven days. During the task participants attempted to produce torque at two set targets without the aid of real-time visual feedback before (Baseline), during (Pain/No Pain), and after (Recovery) the induction of pain and no pain (time factor). Measures of torque, rating of perceived effort (RPE), surface electromyography (sEMG) and heart rate (HR) were taken during each contraction. Pain intensity was recorded continuously using an electronic visual analogue scale (VAS) and pain quality through the completion of a McGill

167 Pain Questionnaire (MPQ). A schematic of the experimental design and protocol is outlined in Figure 1. 

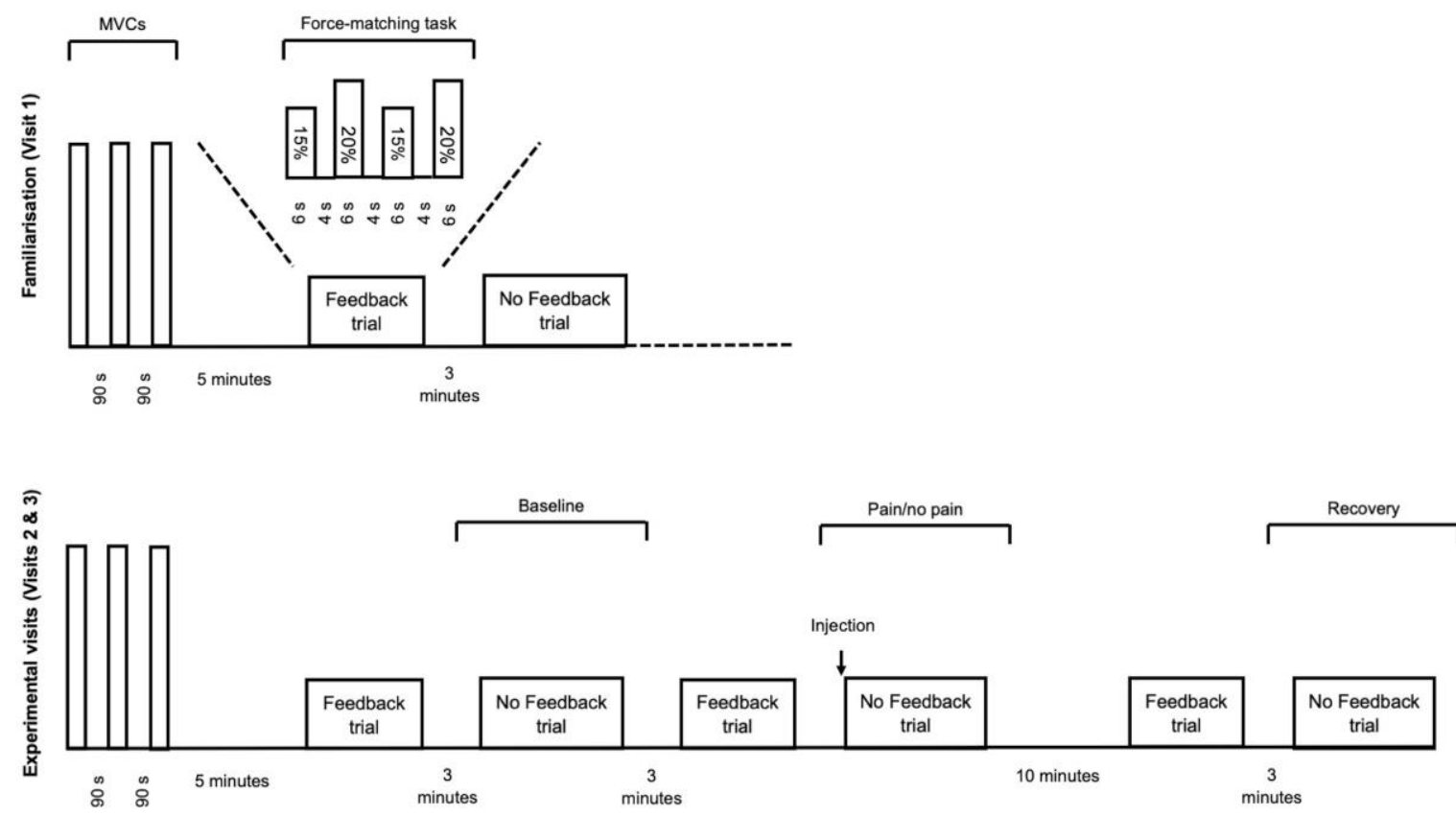

Fig 1. Schematic overview of the experimental design and procedures. MVICs: maximal voluntary contractions

\section{Experimental Procedures}

Torque matching and reproduction task

177 All visits were performed seated on an isokinetic dynamometer (Cybex HUMAC Norm isokinetic dynamometer; CSMi, Soughton, MA, USA) set up for the right leg, with the knee set at an angle of $75^{\circ}$ of flexion $\left(0^{\circ}=\right.$ full extension of the knee $)$, and a hip angle of $90^{\circ}$.

Torque matching and reproduction for knee extension were determined at isometric contractions of $15 \%$ and $20 \%$ maximal voluntary isometric torque (MVIT). These values were selected based on the percentage of MVIT utilised during maximal (100\% maximal oxygen uptake; $\left.\mathrm{VO}_{2 \mathrm{MAX}}\right)$ and submaximal $\left(70 \% \mathrm{VO}_{2 \mathrm{MAX}}\right)$ cycling exercise performed at a pedal rate between $60-80$ revolutions per minute (24). At the start of each visit, participants completed $3 \times 3$ s maximum voluntary isometric contractions (MVICs) separated by $90 \mathrm{~s}$ rest, with the greatest instantaneous value taken as MVIT. If the MVIT of consecutive MVICs 
were not within 5\% of each other, additional MVICs were performed until this criteria was achieved.

Participants attempted the target torques in a trial with real-time torque-production visual feedback ('Feedback Trial') and a trial without visual feedback ('No Feedback Trial').

During the Feedback Trials, target torques (15\% and 20\% MVIT) were presented with actual torque produced via a computer display. Participants were instructed to remember muscular sensations experienced during each target torque and use these to reproduce the same torque in the subsequent No Feedback Trial (7). All Feedback and No Feedback trials were separated by a 3-minute period of rest.

For each trial, participants performed four $6 \mathrm{~s}$ contractions separated by $4 \mathrm{~s}$ of rest in a randomised counter-balanced order, which provided two attempts at both target torques (i.e. $2 \times 15 \%$ MVIT, $2 \times 20 \%$ MVIT). During each contraction, participants were instructed to try and match the target torque within the first $2 \mathrm{~s}$, and then maintain it for a further $4 \mathrm{~s}$.

\section{Intramuscular injection procedure}

A single bolus of $1.0 \mathrm{~mL}$ hypertonic saline (5.8\%) was manually injected into the middle third of the VL of the right leg over a $20 \mathrm{~s}$ window (10 s infusion period). The injection was performed using a $3 \mathrm{~mL}$ Luer-Lok syringe connected to a $25 \mathrm{G} \times 38 \mathrm{~mm}$ SurGuard2 disposable stainless needle (Terumo, Japan). In the control condition, a single bolus of 1.0 $\mathrm{mL}$ isotonic saline $(0.9 \%)$ was injected. 
Participant anthropometric and descriptive measures of age, height, body mass, and hours of physical activity engaged in per week were recorded. Participants were then familiarised with the RPE and pain scales (8), as well as the performance of MVICs, and the Feedback/No Feedback Trials. Five minutes after the completion of the final MVIC, participants performed an initial Feedback Trial followed by a No Feedback Trial. Verbal confirmation of the actual torque produced in each contraction was given after the completion of the trial. All four contractions in the No Feedback Trial were required to be within $10 \%$ of target torque, with further No Feedback Trials completed until this was satisfied. The visit concluded upon the successful completion of a No Feedback Trial or following ten unsuccessful trials.

Visits $2 \& 3-$ Experimental visits

All participants completed a Control (isotonic saline) and an Experimental (hypertonic saline) condition in a randomised and counterbalanced order. In each condition, five-minutes after the completion of the MVICs, participants completed six trials (Feedback, No Feedback, Feedback, No Feedback, Feedback, No Feedback). Prior to the second No Feedback Trial, participants received an intramuscular injection of either isotonic (Control) or hypertonic saline (Experimental), with the No Feedback Trial beginning $20 \mathrm{~s}$ after the removal of the needle. This ensured that the $15 \%$ and $20 \%$ MVIT contractions in this No Feedback Trial were performed with a "moderate" to "strong" muscle pain intensity elicited from the painful hypertonic saline infusion. Ten minutes after the completion of this second No Feedback Trial, the final Feedback and No Feedback (Recovery) Trials were performed. 


\section{Perceptual and psychological measurements}

At the start of each visit participants rated the expected pain $(0=$ "no pain" to $10=$ "worst possible pain") and their confidence to cope with it $(0=$ "not confident at all" to $10=$ “completely confident”). Muscle pain was evaluated by intensity and quality. Participants rated pain intensity on a moment-to-moment basis using an electronic VAS ranging from 0 ("no pain") to 10 ("extremely intense pain"). Participants were instructed to anchor the scale to previous experiences of EIP (4). The device recorded the reported pain value every $5 \mathrm{~s}$, providing measures of pain for each individual contraction. In addition, onset pain intensity (VAS onset), maximal pain intensity (VAS peak), time to maximal intensity (VAS time to peak; from the commencement of sampling), mean pain intensity (VAS mean) and duration of pain (VAS duration; from VAS onset until the state of "no pain") were also calculated using data from the electronic VAS.

After the second No Feedback Trial, when pain had subsided, Total Pain Rating Index and Subclass Rating Index was calculated using a 78 item MPQ (29), with overall quality of pain described by descriptors (sensory, affective, evaluative and miscellaneous) chosen by more than one-third of participants. Upon the completion of each trial, participants provided a RPE, defined as the effort to drive the limb (32), of both target torques using the 15-point Borg (620) scale (6).

\section{Physiological measurements}

Heart rate (HR) was recorded upon the completion of each individual contraction, and muscle electrical activity was continuously recorded using surface electromyography (sEMG). sEMG was attained through square surface electrodes $(\mathrm{Ag} / \mathrm{AgCl}, 32 \times 32 \mathrm{~mm}$; Nessler Medizintechnik, Innsbruck, Austria) mounted in a bipolar set-up, and placed on the muscle 
belly of the VL, vastus medialis (VM) and rectus femoris (RF). For each muscle a reference electrode was placed on the patella. Prior to application of the electrodes, the skin was shaven and cleansed with an alcohol swab. The electrical signal was sampled at $1000 \mathrm{~Hz}$ (Biopac MP150, Biopac Systems Inc., California, USA).

\section{Data analysis}

The sEMG and torque data (for analysis of torque output complexity) were analysed using custom code written in MATLAB 2018a (The MathWorks, Massachusetts, USA).

\section{Torque and error}

Torque was recorded through Spike2 software (Cambridge Electronics Design (CED), Cambridge, UK). For each $6 \mathrm{~s}$ contraction, the torque produced over the last $4 \mathrm{~s}$ was averaged. The average of the actual torque produced for each $15 \%$ and $20 \%$ target was used to define the error in participant torque reproduction. Error was defined as the unidirectional difference between the required target torque and the actual torque produced, and expressed as a percentage of MVIT (i.e. actual torque of $17.5 \%$ MVIT for the $15 \%$ MVIT target would be equal to an error of $2.5 \%$ MVIT). All values of error are presented as positive integers regardless of whether the participant over- or undershot the target torque. The pain on the VAS reported for the corresponding contractions were also averaged for the two attempts at each target torque to provide a mean VAS value for each target torque.

\section{Surface electromyography (sEMG)}

To create a linear envelope representation of the data, rectified absolute values of the raw sEMG signals were two-pass zero-lag filtered using a fourth-order low-pass Butterworth filter, with a cut-off frequency of $5 \mathrm{~Hz}$. The amplitude for the VL, RF and VM were averaged 
over the final $4 \mathrm{~s}$ period of each $6 \mathrm{~s}$ contraction. These values were normalised to the maximum amplitude of the prior MVICs (\% MVIC). For each trial, the sEMG activity was averaged for the two contractions performed at each target torque.

\section{Torque complexity}

The complexity and regularity of the torque output was estimated through the use of approximate entropy (ApEn) and sample entropy (SampEn) $(37,43)$. When applied to physiological time-series data, ApEn is an index that quantifies the predictability or probability of the subsequent values based on prior values, whilst SampEn provides the same output but excludes self-matches $(37,43)$. Both ApEn and SampEn are defined by a value between 0 ('high regularity, low complexity') and 2 ('low regularity, high complexity'). A detailed guide to the algorithms for the calculation of ApEn are evidenced in the appendix of Slifkin and Newell (48), whilst SampEn was calculated using the parameters outlined by Pethick and colleagues (34).

\section{Statistical analysis}

To compare reproduction error between the Control and Experimental conditions at the three time-points (Baseline, Pain/No Pain, and Recovery), a Levene's test was used to determine equality of variance for each normalised target torque (15\% and 20\% MVIT). Changes in HR, RPE, sEMG activity and complexity were evaluated using two-way Analysis of variance (ANOVA) with treatment factor with two fixed levels (Control, Experimental) and a repeated measures Time factor with two time-points (Baseline, Pain/No Pain). A two-way ANOVA with a treatment factor with two fixed levels (No Feedback, Feedback) and a repeated measures Time factor with two time-points (Baseline, Pain/No Pain) was also implemented to evaluate changes in complexity. When an interaction effect was observed, follow-up paired 
samples t-tests were used to assess differences between conditions. Paired samples t-tests were also implemented to evaluate the differences between conditions for pain expectation and confidence, VAS scores, pre-test PANAS, and the change in torque produced in Baseline compared to the Pain/No Pain time-point. A Pearson Bivariate correlation was used to evaluate the correlation between torque error and VAS score reported during the Pain/No Pain contractions. Cohen's guidelines of 0.1 (small), 0.3 (medium) and greater than or equal to 0.5 (large) were used to indicate the strength of correlation.

All data was checked for the standard assumptions associated with the performance of the above statistical tests prior to analysis. Data that did not satisfy the Shapiro-Wilk test of normality $(P<0.05)$ were logarithmically transformed. Results are presented as mean \pm standard deviation (SD). Cohen's $d$ and partial eta square $\left(\eta_{\mathrm{p}}^{2}\right)$ values are reported as measures of effect size. Statistical significance was accepted at an alpha level of $P<0.05$. All statistical analysis were completed using SPSS Statistics v25.0 (SPSS, IBM, New York, USA).

\section{RESULTS}

\section{Experimental muscle pain}

As shown in Table 1, paired samples t-tests revealed a significant difference in VAS pain data between the Control and Experimental conditions. The pain experienced in Experimental was significantly greater in terms of the onset VAS pain reported, with a significantly longer time to peak, yet greater peak VAS pain compared to Control. The reported VAS pain in Experimental was also longer in duration, inducing a significantly greater mean VAS pain, equivalent to a "moderate" to "somewhat strong" muscle pain, and therefore producing a greater overall VAS pain area than Control. 
338 The pain experienced in Experimental was predominantly described in the sensory and

339

340

341

342

343

344

\begin{tabular}{llll}
\hline & Control & Experimental & $P$ \\
\hline VAS mean & $0.8 \pm 1.0$ & $3.1 \pm 1.0^{* *}$ & $<0.001$ \\
VAS peak & $1.6 \pm 2.2$ & $5.7 \pm 1.7^{* *}$ & $<0.001$ \\
VAS onset & $0.5 \pm 0.8$ & $1.7 \pm 1.3^{*}$ & 0.012 \\
VAS time to peak (s) & $41 \pm 29$ & $71 \pm 24^{*}$ & 0.020 \\
VAS duration (s) & $55 \pm 56$ & $233 \pm 60^{* *}$ & $<0.001$ \\
VAS area & $86.3 \pm 115.4$ & $759.8 \pm 325.6^{* *}$ & $<0.001$
\end{tabular}

Table 1. Summary VAS pain data across the entire duration of the Control and Experimental

conditions evaluative dimensions of pain as "aching" (50\% of participants), "throbbing" (43\% of participants), "shooting” (36\% of participants), “cramping" (36\% of participants), "annoying” (36\% of participants). This produced a mean Total Pain Index of $14 \pm 8$, with an overall Present Pain Intensity of $2.1 \pm 0.7$ (“discomforting”).

During the Pain/No Pain trial, a paired samples t-test revealed no significant difference $\left(t_{13}=-\right.$ $0.9, P=0.366, \mathrm{CI} .95-0.9,0.3, d=0.1)$ in mean VAS between contractions performed at $15 \%$ MVIT (4.2 \pm 1.9$)$ and 20\% MVIT (4.5 \pm 2.2$)$ in the Experimental condition. Each of the two target torques in the Pain/No Pain trial was therefore completed at a similar intensity of pain (Fig 2b. and Fig 3b.).

Values are means \pm SD. ${ }^{* *}$ Significant difference between Control and Experimental $(P<$ (no pain) to 10 (extremely intense pain) 

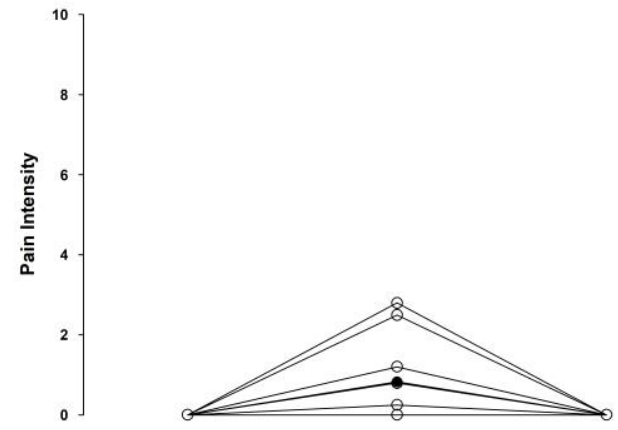

$\theta$ Control

c

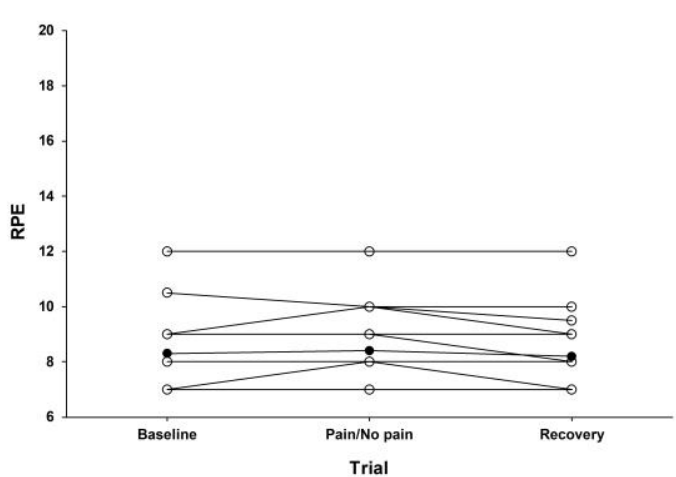

b

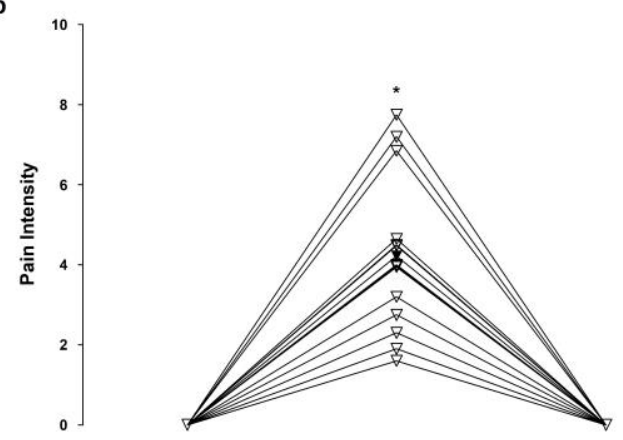

$\nabla$ Experimental

d

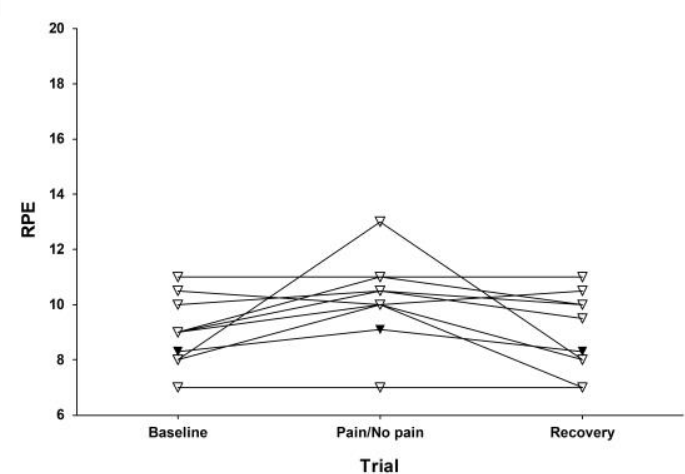

357 Fig 2. Individual (open symbol) and group mean (filled symbol) perceptual differences between conditions (Control and Experimental) at Baseline, Pain/No Pain and Recovery

359 time-points at a target torque of 15\% MVIT. Differences in pain intensity after injection of 360 isotonic saline (Control, $a$ ) and hypertonic saline (Experimental, $b$ ). Differences in RPE in 361 Control $(c)$ and Experimental $(d)$ conditions. *Significantly greater where hypertonic saline was injected. 

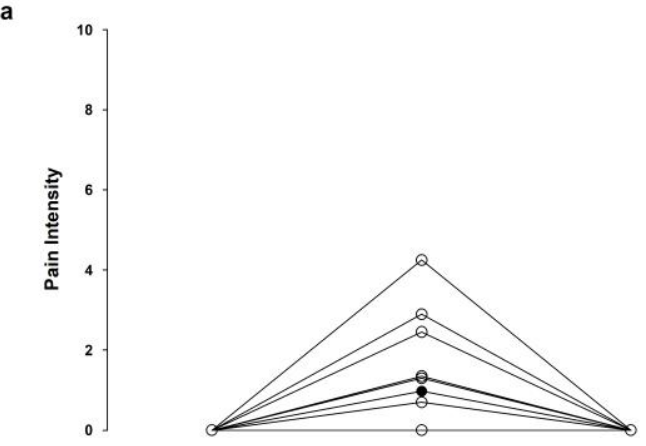

$\ominus$ Control

c

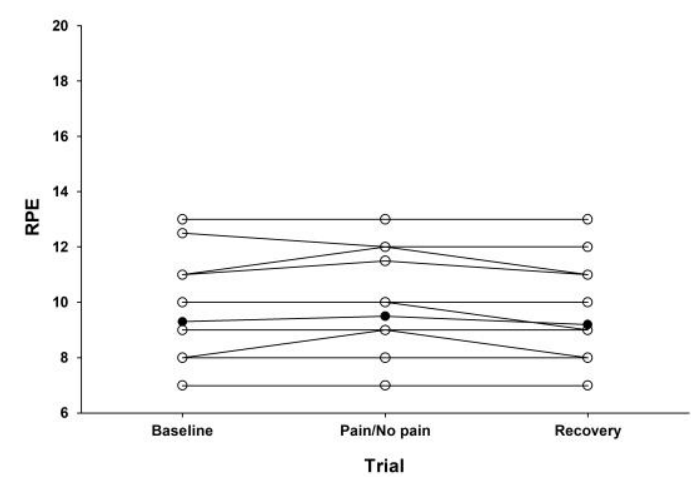

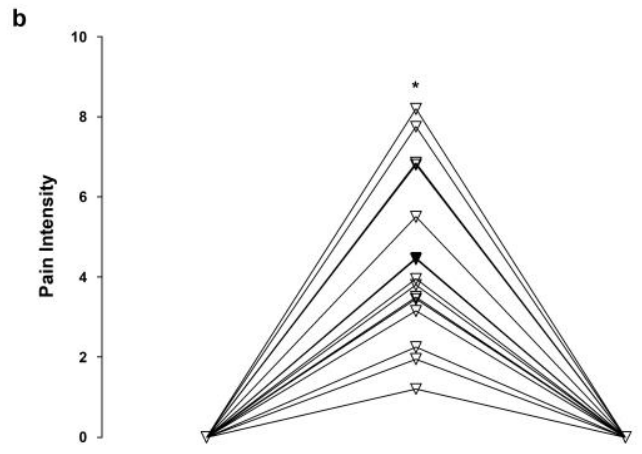

$\nabla$ Experimental

d

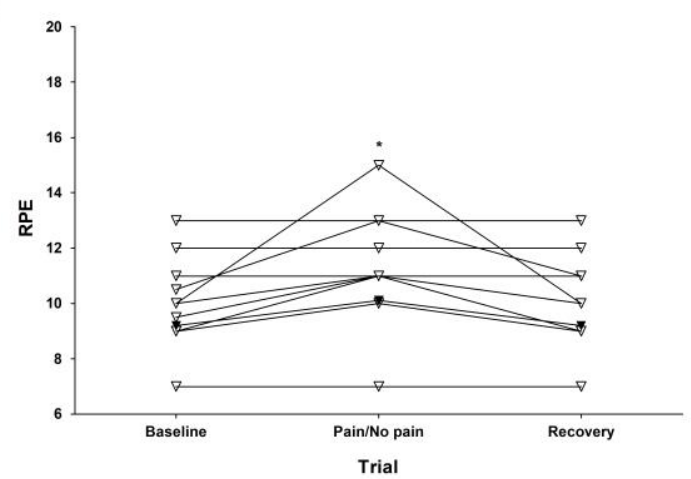

371 Fig 3. Individual (open symbol) and group mean (filled symbol) perceptual differences

372

373

374

between conditions (Control and Experimental) at Baseline, Pain/No Pain and Recovery

time-points at a target torque of $20 \%$ MVIT. Differences in pain intensity after injection of isotonic saline (Control, $a$ ) and hypertonic saline (Experimental, $b$ ). Differences in RPE in Control $(c)$ and Experimental $(d)$ conditions. *Significantly greater where hypertonic saline was injected

Paired samples $t$ tests revealed no significant difference $\left(t_{13}=-1.8, P=0.096, \mathrm{CI} .95-2.08,0.19\right.$, $d=0.5)$ in expectations of pain between the Control $(4.5 \pm 2.1)$ and Experimental $(5.4 \pm 1.8)$ conditions, with no significant differences in the confidence to cope with the expected pain 
$\left(t_{13}=0.2, P=0.818, \mathrm{CI}_{95}-0.29,0.37, d=0.1\right)$ between Control $(9.5 \pm 1.0)$ and Experimental $(9.4$ $\pm 1.0)$.

\section{Comparisons of torque production accuracy}

386 In the presence of greater levels of pain, participants demonstrated an increased variability in their ability to reproduce target torque without visual feedback. However, once the pain had subsided, participants were able to produce the target torque with the same accuracy as Baseline. This is demonstrated by the Levene test for equality of variance, which revealed a significant difference in the variance of mean contraction torque in the Pain/No Pain trial between the Experimental and Control conditions at both $15 \%$ MVIT $\left(F_{1,26}=4.3, P=0.049\right.$, $d=0.6)$ and $20 \%$ MVIT $\left(F_{1,26}=12.0, P=0.002, d=1.0\right)$, as shown in Figures 4 and 5. There was no correlation between Pain/No Pain error and the pain intensity reported during the contractions $(15 \%$ MVIT; $r=-0.053, P=0.858,20 \%$ MVIT; $r=0.172, P=0.557)$. In addition, there was no significant difference in variance between conditions at the Baseline $(15 \%$ MVIT; $F_{1,26}=0.2, P=0.612, d=0.1,20 \%$ MVIT; $\left.F_{1,26}=2.1, P=0.161, d=0.2\right)$ and Recovery $\left(15 \%\right.$ MVIT; $F_{1,26}=1.8, P=0.195, d=0.2,20 \%$ MVIT; $\left.F_{1,26}=3.9, P=0.058, d=0.4\right)$ time-points. 

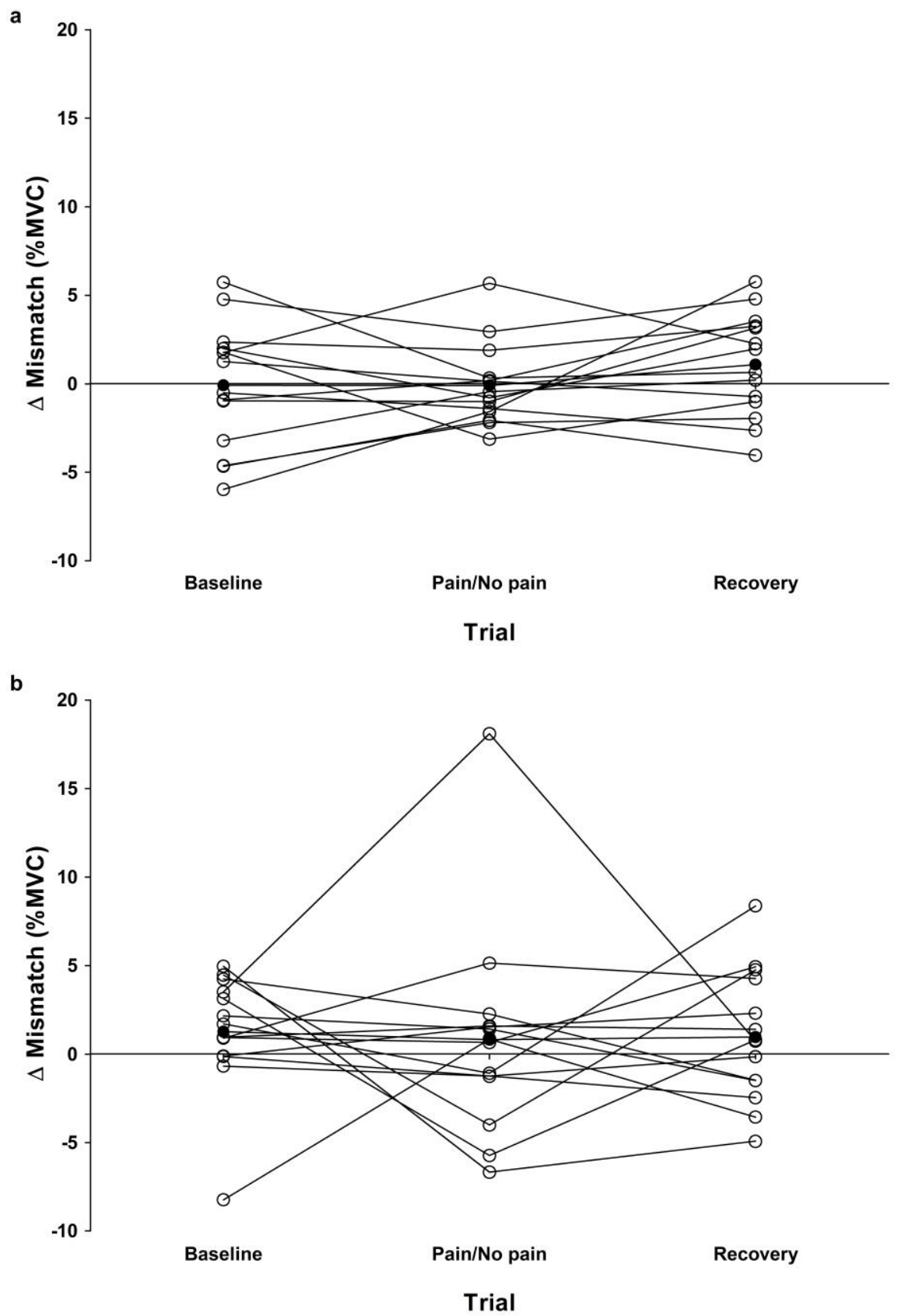

400 Fig 4. Individual (open circle) and group mean (filled circle) torque reproduction error at a 401 target torque of 15\% MVIT before (Baseline), during (Pain/No Pain) and after (Recovery) 402 injection of isotonic saline (Control, $a$ ) or hypertonic saline (Experimental, $b$ ). 

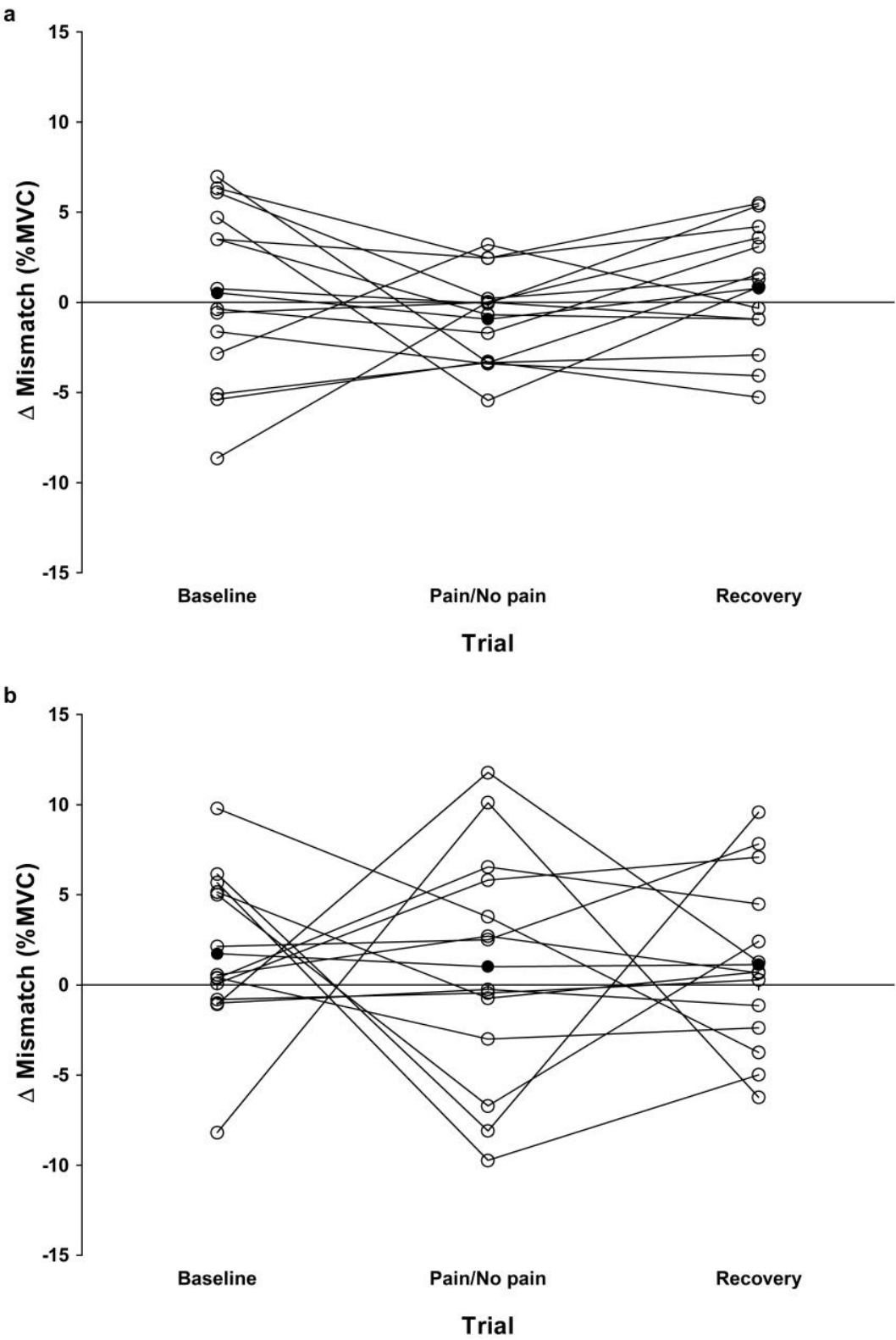

Fig 5. Individual (open circle) and group mean (filled circle) torque reproduction error at a target torque of 20\% MVIT before (Baseline), during (Pain/No Pain) and after (Recovery) injection of isotonic saline (Control, $a$ ) or hypertonic saline (Experimental, $b$ ).

413 A paired samples t-test found no significant difference in the change in torque mismatch 414 between Baseline and Pain/No Pain trials at 15\% MVIT $\left(t_{13}=-1.5, P=0.169, \mathrm{CI}_{.95}-1.1,0.2\right.$, $d=0.5)$ when comparing the Control $(2.5 \pm 1.7 \%$ MVIT $)$ and Experimental $(4.8 \pm 4.8$ 
\%MVIT) conditions. Furthermore, the paired samples t-test highlighted no significant difference in the same change in torque mismatch between Control $(4.2 \pm 3.5 \% \mathrm{MVIT})$ and Experimental $(7.4 \pm 6.0 \%$ MVIT $)$ when contractions were performed at $20 \%$ MVIT $\left(t_{13}=-1.3\right.$, $\left.P=0.235, \mathrm{CI}_{95}-1.6,0.4, d=0.4\right)$. This suggests that the target torque absolute error in the 'Pain/No Pain' was similar to the error made at Baseline despite the change in pain experienced.

Rating of perceived effort

It was apparent that the effort experienced during the contraction was greater in the presence of increased pain, when performed at 20\% MVIT. The $2 \times 2$ (condition $\times$ trial) repeated measures ANOVA demonstrated a significant interaction effect at 20\% MVIT for RPE over trials between conditions $\left(F_{1,13}=6.0, P=0.030, \mathrm{\eta}_{\mathrm{p}}{ }^{2}=0.314\right)$. Follow-up paired samples t-tests revealed a significantly greater $\operatorname{RPE}\left(t_{13}=-2.3, P=0.038, \mathrm{CI}_{95}-1.31,-0.04, d=0.3\right)$ in the Pain/No Pain trial in Experimental compared to Control. A significantly greater $\left(t_{13}=-2.4\right.$, $\left.P=0.033, \mathrm{CI}_{95} 0.1,1.8, d=0.4\right) \mathrm{RPE}$ was also reported in the Experimental condition at the Pain/No Pain trial compared to the Baseline trial. No significant main effect of condition was observed at either 15 or $20 \%$ MVIT $(P>0.05)$. A significant effect of trial was reported at

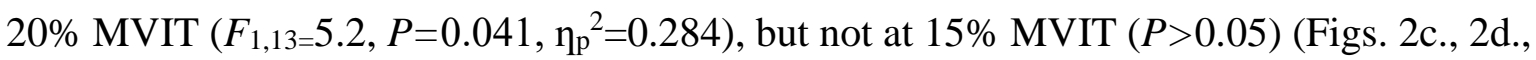
3c. and 3d.). There was no interaction effect observed at 15\% MVIT ( $P>0.05)$.

Surface electromyography (sEMG)

Due to excessive noise in sEMG signal, two participants were removed from the dataset and analysis was performed on the remaining participants $(n=12)$. Despite a greater variance in mean contraction torque in the presence of muscle pain, there were no discernible alterations in activation of the agonist and synergist muscles. At 15 and 20\% MVIT, the performance of 
441 a 2 x 2 (condition $\mathrm{x}$ trial) repeated measures ANOVA demonstrated no significant main effect 442 of condition or trial in either the VL, VM or RF $(P>0.05)$. The VL, VM or RF also demonstrated no significant interaction effect for sEMG activity over trial between conditions 444 at both target torques $(P>0.05)$.

Torque complexity

447 As shown in Table 2, the presence of visual feedback resulted in a more complex (less regular) torque signal (assessed by both ApEn and SampEn) than when torque was being reproduced (No Feedback Trials) $(P<0.001)$. No condition $(P>0.05)$ and no interaction effect was observed for either ApEn or SampEn $(P>0.05)$ at both target torques. At 15 and $20 \%$ MVIT, the performance of a $2 \times 2$ (condition $\times$ trial) repeated measures ANOVA demonstrated no significant main effect of condition for either ApEn or SampEn, as well as no significant main effect of trial for either complexity statistic $(P>0.05)$. There was no interaction effect observed for either ApEn or SampEn $(P>0.05)$ at both target torques.

Heart rate $(H R)$

The $2 \times 2$ (condition $\mathrm{x}$ trial) repeated measures ANOVA revealed no significant main effect of condition at 15 or $20 \%$ MVIT $(P>0.05)$. At $15 \%$ MVIT there was no significant main

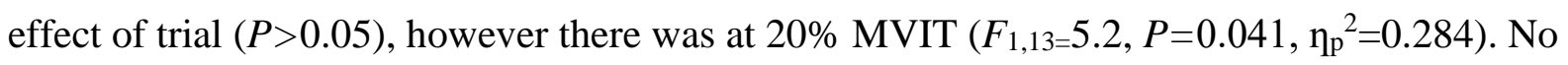
significant interaction effect for HR and trial between conditions was observed at 15 or $20 \%$ MVIT $(P>0.05)$. 
Table 2. Torque complexity (ApEn) during Feedback and No Feedback trials at the Baseline and Pain/No Pain time-points

\begin{tabular}{|c|c|c|c|c|c|c|}
\hline \multirow[t]{3}{*}{ Condition } & \multirow[t]{3}{*}{ Time-point } & \multirow[t]{3}{*}{ Trial } & \multicolumn{4}{|c|}{ Target Torque } \\
\hline & & & \multicolumn{2}{|c|}{$15 \%$ MVIT } & \multicolumn{2}{|c|}{$20 \%$ MVIT } \\
\hline & & & ApEn & SampEn & ApEn & SampEn \\
\hline \multirow[t]{4}{*}{ Control } & Baseline & Feedback & $\begin{array}{l}0.71 \pm \\
0.25^{*}\end{array}$ & $\begin{array}{l}0.71 \pm \\
0.29 *\end{array}$ & $\begin{array}{l}0.57 \pm \\
0.22 *\end{array}$ & $\begin{array}{l}0.56 \pm \\
0.27^{*}\end{array}$ \\
\hline & & $\begin{array}{l}\text { No } \\
\text { Feedback }\end{array}$ & $\begin{array}{l}0.35 \pm \\
0.17 *\end{array}$ & $\begin{array}{l}0.32 \pm \\
0.17 *\end{array}$ & $\begin{array}{l}0.31 \pm \\
0.21^{*}\end{array}$ & $\begin{array}{l}0.29 \pm \\
0.22 *\end{array}$ \\
\hline & $\begin{array}{l}\text { Pain/No } \\
\text { Pain }\end{array}$ & Feedback & $\begin{array}{l}0.73 \pm \\
0.21 *\end{array}$ & $\begin{array}{l}0.72 \pm \\
0.24 *\end{array}$ & $\begin{array}{l}0.60 \pm \\
0.26^{*}\end{array}$ & $\begin{array}{l}0.61 \pm \\
0.30 *\end{array}$ \\
\hline & & $\begin{array}{l}\text { No } \\
\text { Feedback }\end{array}$ & $\begin{array}{l}0.35 \pm \\
0.21^{*}\end{array}$ & $\begin{array}{l}0.32 \pm \\
0.22 *\end{array}$ & $\begin{array}{l}0.28 \pm \\
0.17^{*}\end{array}$ & $\begin{array}{l}0.26 \pm \\
0.17^{*}\end{array}$ \\
\hline \multirow[t]{4}{*}{ Experimental } & Baseline & Feedback & $\begin{array}{l}0.78 \pm \\
0.24^{*}\end{array}$ & $\begin{array}{l}0.79 \pm \\
0.30^{*}\end{array}$ & $\begin{array}{l}0.64 \pm \\
0.21 *\end{array}$ & $\begin{array}{l}0.64 \pm \\
0.25^{*}\end{array}$ \\
\hline & & $\begin{array}{l}\text { No } \\
\text { Feedback }\end{array}$ & $\begin{array}{l}0.29 \pm \\
0.13^{*}\end{array}$ & $\begin{array}{l}0.26 \pm \\
0.13^{*}\end{array}$ & $\begin{array}{l}0.27 \pm \\
0.12^{*}\end{array}$ & $\begin{array}{l}0.24 \pm \\
0.12^{*}\end{array}$ \\
\hline & $\begin{array}{l}\text { Pain/No } \\
\text { Pain }\end{array}$ & Feedback & $\begin{array}{l}0.74 \pm \\
0.27^{*}\end{array}$ & $\begin{array}{l}0.75 \pm \\
0.31 *\end{array}$ & $\begin{array}{l}0.68 \pm \\
0.23^{*}\end{array}$ & $\begin{array}{l}0.68 \pm \\
0.28^{*}\end{array}$ \\
\hline & & $\begin{array}{l}\text { No } \\
\text { Feedback }\end{array}$ & $\begin{array}{l}0.32 \pm \\
0.19^{*}\end{array}$ & $\begin{array}{l}0.29 \pm \\
0.19^{*}\end{array}$ & $\begin{array}{l}0.22 \pm \\
0.11^{*}\end{array}$ & $\begin{array}{l}0.20 \pm \\
0.10^{*}\end{array}$ \\
\hline
\end{tabular}
within condition and time-point $(\mathrm{P}<0.001)$. 


\section{DISCUSSION}

475 The present study demonstrates for the first time that the experience of muscle pain, administered by the intramuscular injection of hypertonic saline into the VL, resulted in a

477 greater variance in the mean contraction torque at both 15 and 20\% MVIT when compared to 478 the injection of isotonic saline (a placebo control). The increased variance was paralleled by an elevated experience of pain at both contraction intensities, and a greater perceived effort when performed at 20\% MVIT. Once the pain had subsided, accuracy of torque production returned to baseline levels. This study for the first time demonstrates that the presence of muscle pain (that feels like EIP) impedes the ability to accurately reproduce torque in the knee extensors. This important finding provides key experimental evidence for the deleterious implications of EIP on the ability to self-regulate exercise intensity.

Effect of pain on isometric torque reproduction

The purpose of the present study was to establish whether the presence of pain in a muscle with a major contributing role to force generation during both dynamic contractions and whole-body exercise (i.e. the VL) has a debilitative effect on producing a given torque using the ipsilateral knee extensor muscle group. The primary finding from this study is that the mismatch between the actual torque produced and the target torque (when required to reproduce both 15 and $20 \%$ MVIT) was significantly more variable with pain, with no discernible direction of error (i.e. participants both under- and overshot the target torque). Resultantly, this study is the first to demonstrate that the experimental induction of pain in a large locomotor muscle group impairs the judgement of torque during an isometric reproduction task performed at an intensity of relevance to endurance exercise performance. 
The compromised ability to accurately reproduce torque during pain is in line with previous research that has implemented the hypertonic saline model in the elbow flexors to investigate the impact of pain on estimation error in a contralateral torque estimation task $(40,41,57)$. However, this prior literature has consistently reported that participants specifically overestimated the torque that is produced in the painful muscle, and therefore produced less torque than required. In contrast with lack of direction in error reported in the present study, this observed disparity could be due to potential differences in the limb evaluated (e.g. contralateral or ipsilateral). Alternatively, as the knee extensor muscles respond differently to exercise-induced fatigue (55), the muscle group tested (elbow flexor vs. knee extensors) should also be considered.

\section{Proposed mechanisms}

The presence of the hypertonic saline solution in addition to the short-duration muscle contraction creates a noxious environment within the skeletal musculature (31), which results in an alteration in activity of both ascending metaborecptive and nociceptive group III and IV afferent fibers (18). In this noxious environment, there are several neuromuscular mechanisms that, when acting in singularity or in combination, may provide an explanation for the impaired reproduction of torque in the present study.

Convergent projection from group III and IV afferents on common interneurons from group Ib proprioceptive afferents (45) provide information on muscle force (15). As discussed by Salomoni and Graven-Nielsen (44), the large variance in the mean contraction torque in the Experimental condition could be a result of the spatial facilitation between these afferents interfering in the central interpretation of proprioceptive information essential for the accurate control of torque. A discrepancy between the centrally mediated judgement of 
torque and the actual afferent feedback from the periphery could therefore have resulted in the torque reproduction error.

In addition, the projection of the group III and IV afferents have inhibitory effects on the central nervous system. The increased afferent feedback from the hypertonic saline may have limited motor cortical excitability, and reduced central motor drive and voluntary activation of the knee extensors $(14,19)$. In order to compensate for the hypertonic saline-induced impairment of motor cortex excitability, a greater effort is required to drive the limb to meet the required torque $(30,39)$. As an outcome reflected in the present study, this could provide a possible explanation for some of the differences in actual and perceived torque produced. The findings from Proske and colleagues (40) where the matching of torque through effort resulted in an overshoot of the target torque, are in support of this explanation.

Despite the observed impairment in torque-reproduction performance during pain, there was no change in the torque complexity of the knee extensors, or the level of muscle activity assessed by sEMG. The absence of alterations in sEMG is comparable with findings from the established literature into the implications of EIP on muscle activity during submaximal isometric contractions, where a lack of marked changes in sEMG signal are also observed $(16,44,46)$. Combined, these observations contradict the underpinning theory of the 'Pain Adaptation Model' (25) where it is predicted that the presence of pain has a reliable inhibitory influence on agonist muscles, whilst simultaneously activating the antagonists. Instead, the observations of the present study could, with caution, be in-line with the "moving differently in pain" model proposed by Hodges and Tucker (17). This theory postulates that pain initiates a non-uniform effect across the motor neurone pool, causing a redistribution of activity between and within muscles to provide a key adaptive and protective function. Whilst 
this alteration has the immediate benefit of minimising the pain experienced and preventing further injury or damage to the area in pain during muscular contraction, this change to a "sub-optimal" movement strategy could have consequences for the efficiency of task performance $(17,53)$. Detection of these adaptations would however require the use of finewire electrodes (52) or high density sEMG, as a combination of changes in order of motor unit activation or synchronisation can occur without alteration in amplitude of gross sEMG (51).

A loss of knee-extensor torque complexity during both prolonged maximal and submaximal contractions has been closely associated with fatigue $(34,35)$, and is suggested to have a detrimental impact on the performance of motor tasks in the lower limb (10). In the present study, the lack of change in torque complexity suggests that the acute pain from the hypertonic saline was unlikely to have independently caused neuromuscular fatigue. The increased variance in mean contraction torque is therefore unable to be explained by paininduced mechanisms of fatigue during the short-duration and submaximal isometric contractions.

This finding is consistent with prior literature, where differences in torque complexity are not observed in the first few seconds of isometric muscle contraction despite the presence of pain (from an eccentric contraction muscle damage protocol) and the consequential impaired ability to perform a maximal voluntary contraction (33). As torque complexity progressively decreases over time during submaximal contractions until the point of task failure (34), if the torque reproduction task in the present study was performed over a longer duration, a paininduced acceleration of exercise-induced fatigue (and therefore loss of torque complexity) would likely be observed in addition to the impaired the ability to accurately reproduce 
torque. As such the findings of the present study reinforce the notion that acute, moderate muscle pain alone is not necessarily fatiguing, but may accelerate the development of fatigue during prolonged or exhaustive exercise $(27,50)$, or impair maximal voluntary contraction.

A further point of consideration is that in the absence of visual feedback, and sole reliance on afferent/efferent information and task memory, the ability to accurately reproduce torque depreciates (22) and that this is characteristically coupled with a lower complexity of the torque signal (indicative of a reduced adaptability in force control) $(21,49)$. This observation is replicated in the present study, and it is noteworthy that the values for ApEn and SampEn in the No Feedback conditions are similar to those shown at task failure in exhaustive exercise (34). Therefore, it is possible that the induction of muscle pain in the present study was not able to reduce the complexity of the torque signal beyond that already caused by the removal of visual feedback.

Alternatively, the compromised ability to accurately reproduce torque (despite no change in loss of torque complexity) could be due to the experience of pain preventing some attentional focus on the task (23), making the task more challenging. It is plausible that the elevated intensity of pain (induced by the injection of hypertonic saline), which was rated as "moderate" to "somewhat strong" in both target torques, provided a stimulus which was perceived as threatening. With some attentional resources focused on coping with the 'threat' of the noxious stimuli, attention may have been directed away from the task, which could have resulted in a compromised accuracy of torque reproduction (11); a notion supported by evidence from previous experimental work $(5,26)$. However, in the current study, there was no relationship between pain intensity and error, which indicates that the sensation of pain alone was unlikely to have had a direct influence on task performance. 
600 Overall, it is evident that the presence of pain interferes with proprioception during

601 submaximal isometric contractions in the lower-limb. The design and findings of the present

602 study therefore provide a key indication of the potential mechanism underpinning the

603 detrimental effect of EIP on exercise intensity regulation and endurance performance. Some

604 caution should however be taken when extrapolating these findings to whole-body exercise.

605 In order to improve task relevance to whole-body locomotor exercise and further apply the

606 findings of the present study, there is the need for the impact of this experimental model to be

607 evaluated during isokinetic or dynamic muscular contractions performed at a varying or

608 higher work rate.

609

610

Methodological considerations

611 Whilst there is inconsistent evidence for sex-related differences in the pain intensity response

612 to the hypertonic saline model $(20,42)$, the fluctuations in hormone concentration across the

613 different menstrual cycle phases may cause differences in pain perception to experimental

614 pain (47). It is acknowledged that the present study did not account for menstrual cycle

615 phases of the female participant, and this is a limitation. It is also important to note that the

616 short-duration and submaximal isometric contractions used in the current study were not

617 fatiguing, and this limits the ability to examine the notion that pain accelerates the

618 development of exercise-induced fatigue in addition to the impairment in accurate torque

619 reproduction. To explore this in combination, future investigations should attempt to employ

620 a similar study design examining torque reproduction ability in the presence of muscle pain

621 during contractions performed at a greater exercise intensity, or over a longer duration.

622

623 Conclusion 
624 In conclusion, the injection of hypertonic saline into the VL during a torque reproduction task 625 created muscle pain that resulted in an impaired ability to accurately produce a given

626 submaximal target torque during a short, submaximal isometric contractions. The presence of 627 pain was linked with a greater effort to drive the limb and meet the given target torque when 628 attempting to contract at $20 \%$ MVIT, but not at $15 \%$ MVIT. The compromised ability to 629 reproduce torque returned to baseline levels once pain had subsided. These findings have 630 implications for the impact of EIP on self-selected work rate regulation during endurance 631 exercise performance.

632

633

634

635

636

637

638

639

640

641

642

643

644

645

646

647

648 


\section{REFERENCES}

654 1. Adamo DE, Scotland S, Martin BJ. Asymmetry in grasp force matching and sense of 655 effort. Exp Brain Res 217: 273-285, 2012. doi: 10.1007/s00221-011-2991-6.

2. Amann M, Blain GM, Proctor LT, Sebranek JJ, Pegelow DF, Dempsey JA. Implications of group III and IV muscle afferents for high-intensity endurance exercise performance in humans. J Physiol 589: 5299-5309, 2011. doi: 10.1113/jphysiol.2011.213769.

660

3. Amann M, Proctor LT, Sebranek JJ, Pegelow DF, Dempsey JA. Opioid-mediated muscle afferents inhibit central motor drive and limit peripheral muscle fatigue development in humans. J Physiol 587: 271-283, 2009. doi: 10.1113/jphysiol.2008.163303.

4. Astokorki AHY, Mauger AR. Transcutaneous electrical nerve stimulation reduces exercise-induced perceived pain and improves endurance exercise performance. Eur J Appl Physiol 117: 483-492, 2017. doi: 10.1007/s00421-016-3532-6.

5. Bennell K, Wee E, Crossley K, Stillman B, Hodges P. Effects of experimentallyinduced anterior knee pain on knee joint position sense in healthy individuals. J Orthop Res 23: 46-53, 2005. doi: 10.1016/j.orthres.2004.06.008.

6. Borg GA. Borg's Perceived Exertion and Pain Scales. Champaign, IL, Human Kinetics, 1998

672 7. Carson RG, Riek S, Shahbazpour N. Central and peripheral mediation of human force sensation following eccentric or concentric contractions. J Physiol 539: 913-925, 
2002. doi: 10.1013/jphysiol.2001.013385.

675

676

677

678

679

680

681

682

683

684

685

686

687

688

689

690

691

692

693

694

695

696

697

698

8. Cook DB, O’Connor PJ, Eubanks SA, Smith JC, Lee M. Naturally occurring muscle pain during exercise: assessment and experimental evidence. Med Sci Sports Exerc 29: 999-1012, 1997. doi: 10.1097/00005768-199708000-00004.

9. Cook DB, O’Connor PJ, Oliver SE, Lee Y. Sex differences in naturally occurring leg muscle pain and exertion during maximal cycle ergometry. Int J Neurosci 95: 183202, 1998. doi: 10.3109/00207459809003340.

10. Cortes N, Onate J, Morrison S. Differential effects of fatigue on movement variability. Gait Posture 39: 888-893, 2014. doi: 10.1016/j.gaitpost.2013.11.020.

11. Eccleston C, Crombez G. Pain demands attention: A cognitive-affective model of the interruptive function of pain. Psychol Bull 125: 356-366, 1999. doi: 10.1037/00332909.125.3.356.

12. Edwards AM, Polman RCJ. Pacing and awareness: Brain regulation of physical activity. Sport Med 43: 1057-1064, 2013. doi: 10.1007/s40279-013-0091-4.

13. Faul F, Erdfelder E, Lang A-G, Buchner A. G*Power 3: A flexible statistical power analysis program for the social, behavioral, and biomedical sciences. Behav Res Methods 39: 175-191, 2007. doi: 10.3758/BF03193146.

14. Gandevia SC. Spinal and Supraspinal Factors in Human Muscle Fatigue. Physiol Rev 81: 1725-1789, 2001. doi: 10.1152/physrev.2001.81.4.1725.

15. Gandevia SC, Burke D. Does the nervous system depend on kinesthetic information to control natural limb movements? Behav Brain Sci 15: 614-632, 1992. doi: 10.1017/S0140525X0007254X.

16. Graven-Nielsen T, Svensson P, Arendt-Nielsen L. Effects of experimental muscle pain on muscle activity and co-ordination during static and dynamic motor function. Electroencephalogr Clin Neurophysiol - Electromyogr Mot Control 105: 156-164, 
700

701

702

703

704

705

706

707

708

709

710

711

712

713

714

715

716

717

718

719

720

721

722

723

17. Hodges PW, Tucker K. Moving differently in pain: A new theory to explain the adaptation to pain. Pain 152: S90-S98, 2011. doi: 10.1016/j.pain.2010.10.020.

18. Laursen RJ, Graven-Nielsen T, Jensen TS, Arendt-Nielsen L. The effect of differential and complete nerve block on experimental muscle pain in humans. Muscle and Nerve 22: 1564-1570, 1999.

19. Le Pera D, Graven-Nielsen T, Valeriani M, Oliviero A, Di Lazzaro V, Tonali PA, Arendt-Nielsen L. Inhibition of motor system excitability at cortical and spinal level by tonic muscle pain. Clin Neurophysiol 112: 1633-1641, 2001. doi: 10.1016/S13882457(01)00631-9.

20. Lei J, You HJ. Variation of pain and vasomotor responses evoked by intramuscular infusion of hypertonic saline in human subjects: Influence of gender and its potential neural mechanisms. Brain Res Bull 87: 564-570, 2012. doi:

10.1016/j.brainresbull.2011.11.003.

21. Li K, Marquardt TL, Li ZM. Removal of visual feedback lowers structural variability of inter-digit force coordination during sustained precision pinch. Neurosci Lett 545: 1-5, 2013. doi: 10.1016/j.neulet.2013.04.011.

22. Limonta E, Rampichini S, Cè E, Esposito F. Effects of visual feedback absence on force control during isometric contraction. Eur J Appl Physiol 115: 507-519, 2015. doi: 10.1007/s00421-014-3036-1.

23. Linton SJ, Shaw WS. Impact of Psychological Factors in the Experience of Pain. Phys Ther 91: 700-711, 2011. doi: 10.2522/ptj.20100330.

24. Löllgen H, Graham T, Sjogaard G. Muscle metabolites, force, and perceived exertion bicycling at varying pedal rates. Med Sci Sports Exerc 12: 345-351, 1980. doi: 10.1249/00005768-198025000-00008. 
25. Lund JP, Donga R, Widmer CG, Stohler CS. The pain-adaptation model: a discussion of the relationship between chronic musculoskeletal pain and motor activity. Can J Physiol Pharmacol 69: 683-694, 1991.

26. Matre D, Arendt-Neilsen L, Knardahl S. Effects of localization and intensity of experimental muscle pain on ankle joint proprioception. Eur J Pain 6: 245-260, 2002. doi: 10.1053/eujp.2002.0332.

27. Mauger AR. Factors affecting the regulation of pacing: current perspectives. Open Access J Sport Med 5: 209-214, 2014. doi: 10.2147/OAJSM.S38599.

28. Mauger AR, Jones AM, Williams CA. Influence of acetaminophen on performance during time trial cycling. J Appl Physiol 108: 98-104, 2010. doi: 10.1152/japplphysiol.00761.2009.

29. Melzack R. The McGill Pain Questionnaire: Major properties and scoring methods. Pain 1: 277-299, 1975. doi: 10.1016/0304-3959(75)90044-5.

30. Mulder T, Zijlstra W, Geurts A. Assessment of motor recovery and decline. Gait Posture 16: 198-210, 2002. doi: 10.1016/S0966-6362(01)00157-6.

31. O'Connor PJ, Cook DB. Exercise and Pain: The Neurobiology, Measurement, and Laboratory Study of Pain in Relation to Exercise in Humans [Online]. Exerc Sport Sci Rev 27: 119-166, 1999. https://journals.lww.com/acsmessr/Citation/1999/00270/5_Exercise_and_Pain_The_Neurobiology,.7.aspx.

32. Pageaux B, Angius L, Hopker JG, Lepers R, Marcora SM. Central alterations of neuromuscular function and feedback from group III-IV muscle afferents following exhaustive high-intensity one-leg dynamic exercise. Am J Physiol Integr Comp Physiol 308: R1008-R1020, 2015. doi: 10.1152/ajpregu.00280.2014.

33. Pethick J, Whiteaway K, Winter SL, Burnley M. Prolonged depression of kneeextensor torque complexity following eccentric exercise. Exp Physiol 104: 100-111, 
2019. doi: 10.1113/EP087295.

750

751

752

753

754

755

756

757

758

759

760

761

762

763

764

765

766

767

768

769

770

771

772

773

34. Pethick J, Winter SL, Burnley M. Fatigue reduces the complexity of knee extensor torque fluctuations during maximal and submaximal intermittent isometric contractions in man. J Physiol 593: 2085-2096, 2015. doi: 10.1113/jphysiol.2015.284380.

35. Pethick J, Winter SL, Burnley M. Fatigue reduces the complexity of knee extensor torque during fatiguing sustained isometric contractions. Eur J Sport Sci 19: 13491358, 2019. doi: 10.1080/17461391.2019.1599450.

36. Philippou A, Bogdanis GC, Maridaki M. Neuromuscular dysfunction with the experimental arm acting as its own reference following eccentric and isometric exercise. Somatosens Mot Res 27: 45-54, 2010. doi: 10.3109/08990220.2010.483204.

37. Pincus SM. Approximate entropy as a measure of system complexity. Proc Natl Acad Sci 88: 2297-2301, 1991. doi: 10.1073/pnas.88.6.2297.

38. Pollak KA, Swenson JD, Vanhaitsma TA, Hughen RW, Jo D, Light KC, Schweinhardt P, Amann M, Light AR. Exogenously applied muscle metabolites synergistically evoke sensations of muscle fatigue and pain in human subjects. Exp Physiol 99: 368380, 2014. doi: 10.1113/expphysiol.2013.075812.

39. Proske U, Gandevia SC. The Proprioceptive Senses: Their Roles in Signaling Body Shape, Body Position and Movement, and Muscle Force. Physiol Rev 92: 1651-1697, 2012. doi: 10.1152/physrev.00048.2011.

40. Proske U, Gregory JE, Morgan DL, Percival P, Weerakkody NS, Canny BJ. Force matching errors following eccentric exercise. Hum Mov Sci 23: 365-378, 2004. doi: 10.1016/j.humov.2004.08.012.

41. Proske U, Weerakkody NS, Percival P, Morgan DL, Gregory JE, Canny BJ. Forcematching errors after eccentric exercise attributed to muscle soreness. Clin Exp 
Pharmacol Physiol 30: 576-579, 2003. doi: 10.1046/j.1440-1681.2003.03880.x.

42. Racine M, Tousignant-Laflamme Y, Kloda LA, Dion D, Dupuis G, Choinire M. A systematic literature review of 10 years of research on sex/gender and pain perception - Part 2: Do biopsychosocial factors alter pain sensitivity differently in women and men? Pain 153: 619-635, 2012. doi: 10.1016/j.pain.2011.11.026.

43. Richman JS, Moorman JR. Physiological time-series analysis using approximate entropy and sample entropy. Am J Physiol Heart Circ Physiol 278: H2039-49, 2000. doi: 10.1152/ajpheart.2000.278.6.H2039.

44. Salomoni SE, Graven-Nielsen T. Experimental muscle pain increases normalized variability of multidirectional forces during isometric contractions. Eur J Appl Physiol 112: 3607-3617, 2012. doi: 10.1007/s00421-012-2343-7.

45. Schomburg ED, Steffens H, Kniffki KD. Contribution of group III and IV muscle afferents to multisensorial spinal motor control in cats. Neurosci Res 33: 195-206, 1999. doi: 10.1016/S0168-0102(99)00006-1.

46. Schulte E, Ciubotariu A, Arendt-Nielsen L, Disselhorst-Klug C, Rau G, GravenNielsen T. Experimental muscle pain increases trapezius muscle activity during sustained isometric contractions of arm muscles. Clin Neurophysiol 115: 1767-1778, 2004. doi: 10.1016/j.clinph.2004.03.005.

47. Sherman JJ, LeResche L. Does experimental pain response vary across the menstrual cycle? A methodological review. Am J Physiol Integr Comp Physiol 291: R245-R256, 2006. doi: 10.1152/ajpregu.00920.2005.

48. Slifkin AB, Newell KM. Noise, information transmission, and force variability. J Exp Psychol Hum Percept Perform 25: 837-851, 1999. doi: 10.1037/0096-1523.25.3.837.

49. Slifkin AB, Vaillancourt DE, Newell KM. Intermittency in the control of continuous force production. J Neurophysiol 84: 1708-18, 2000. doi: 10.1152/jn.2000.84.4.1708. 
50. Smith SA, Micklewright D, Winter SL, Mauger AR. Muscle pain induced by hypertonic saline in the knee extensors decreases single-limb isometric time to task failure. Eur J Appl Physiol 120: 2047-2058, 2020. doi: 10.1007/s00421-020-04425-2.

51. Tucker K, Butler J, Graven-Nielsen T, Riek S, Hodges P. Motor Unit Recruitment Strategies Are Altered during Deep-Tissue Pain. J Neurosci 29: 10820-10826, 2009. doi: 10.1523/JNEUROSCI.5211-08.2009.

52. Tucker KJ, Hodges PW. Motoneurone recruitment is altered with pain induced in nonmuscular tissue. Pain 141: 151-155, 2009. doi: 10.1016/j.pain.2008.10.029.

53. Tucker KJ, Hodges PW. Changes in motor unit recruitment strategy during pain alters force direction. Eur J Pain 14: 932-938, 2010. doi: 10.1016/j.ejpain.2010.03.006.

54. Tucker R. The anticipatory regulation of performance: The physiological basis for pacing strategies and the development of a perception-based model for exercise performance. Br J Sports Med 43: 392-400, 2009. doi: 10.1136/bjsm.2008.050799.

55. Vernillo G, Temesi J, Martin M, Millet GY. Mechanisms of fatigue and recovery in upper versus lower limbs in men. Med Sci Sports Exerc 50: 334-343, 2018. doi: 10.1249/MSS.0000000000001445.

56. Watson D, Clark LA, Tellegen A. Development and validation of brief measures of positive and negative affect: The PANAS scales. J Pers Soc Psychol 54: 1063-1070, 1988. doi: 10.1037/0022-3514.54.6.1063.

57. Weerakkody NS, Percival P, Canny BJ, Morgan DL, Proske U. Force matching at the elbow joint is disturbed by muscle soreness. Somatosens Mot Res 20: 27-32, 2003. doi: 10.1080/0899022031000083816. 
824 ADDITIONAL INFORMATION

825 Acknowledgements

826 Thank you to Shane Massey for his dedication and assistance with data collection.

827

828 Disclosures

829 Conflict of interest

830 The authors declare that they have no conflict of interest.

831

$832 \quad$ Funding

833 No funding sources were provided for the present study. This research project did not receive 834 any specific grant from funding agencies in the public, commercial or not-for-profit sectors.

835

836 